\title{
A Retrospective Analysis of the Merit of Iris-pupil Area Ratio as a Predictor of Risk of Myocardial Infarction
}

\author{
Ifechukwuamaka Chinaka, Arun HS Kumar*
}

\begin{abstract}
Background: We have recently reported the utility of iris-pupil area ratio as a sensitive index of borderline changes in sympathetic activity and the stress associated with it in primates and canines. As minimal increase in sympathetic activity can lead to micro vascular constriction, which is often observed in patients suffering from myocardial infarction, we hypothesised here that iris-pupil area ratio will serve as a predictor of risk of myocardial infarction in humans. Materials and Methods: To test our hypothesis, we analysed and compared the digital images of the eyes of celebrities who succumbed to myocardial infarction with that of healthy celebrities. Most recent digital images of the celebrities available in the public database were used for our analysis. Iris-pupil area ratio of both eyes was measured using NIH Image-J software. Results: The iris-pupil area ratio was significantly lower in celebrities who succumbed to myocardial infarction compared to healthy cohorts $(4.875 \pm 1.506 \mathrm{Vs}$. $6.978 \pm 1.499 ; p<0.001)$. To assess the correlation of age with iris-pupil area ratio we performed regression analysis of the data from each of the cohorts. The age Vs iris-pupil area ratio regression analysis in celebrities who succumbed to myocardial infarction had positive correlation (correlation coefficient of 0.06889), while healthy celebrities showed negative correlation (correlation coefficient of 0.03775). Although the correlation between age and iris-pupil area ratio was weak, a higher negative correlation (correlation coefficient of 0.387) was observed in a cohort of 50 to 80 years old healthy celebrities. Conclusion: The lower iris-pupil area ratio observed in celebrities who succumb to myocardial infarction possibly suggests increased sympathetic activity and microvascular constriction, which supports the merit of iris-pupil area ratio as a non-invasive tool in predicting myocardial infarction.
\end{abstract}

Key words: Iris-pupillary ratio, Sympathetic activity, Myocardial infarction, Risk analysis, Risk prediction.

\section{INTRODUCTION}

The incidence of myocardial infarction continues to rise despite the advancements in healthcare. ${ }^{1,2}$ While significant progression has been achieved in the management of myocardial infarction, similar progression to effectively predict and identify patients at the immediate risk of suffering from myocardial infarction are lacking. ${ }^{3-5}$ Although several biochemical, physiological and lifestyle risk factors are know, the utility of these risk factors in predicting immediate episodes of myocardial infarction is limited. ${ }^{2,4-12}$ This is perhaps due to the limitations in our understanding of the causative factors, which trigger myocardial infarction. While microvascular constriction is reported to be a major culprit, the feasibility to measure this parameter in a dynamic scale is technically limited. ${ }^{6,13,14}$ Physiologically microvasculatures are primarily regulated by autonomic nervous system with a balance between sympathetic and para-sympathetic neurons. ${ }^{15-21}$ While it is feasible to biochemically measure the neurotransmitters of sympathetic and para-sympathetic neurons, often the sensitivity of detecting the neurotransmitters is feasible only

Cite this article : Chinaka I, Kumar AHS. A Retrospective Analysis of the Merit of Iris-pupil Area Ratio as a Predictor of Risk of Myocardial Infarction. BEMS Reports. 2019;5(2):23-5. when they are significantly elevated. Hence detection of borderline changes in the concentrations of the neurotransmitters remains a technical challenge.

We have recently reported the utility of iris-pupil area ratio as a sensitive index of borderline changes in sympathetic activity and the stress associated with it in primates and canines. ${ }^{22,23}$ The reliability of irispupil area ratio to reflect borderline changes in sympathetic activity may be clinically useful to assess several clinical conditions associated with microvascular constrictions including myocardial infarction and stroke. Hence in this study we tested the hypothesis that iris-pupil area ratio may be valuable in identifying human patients at risk of suffering from myocardial infarction.

\section{MATERIALS AND METHODS}

For this study we opted to analyse the digital images of celebrities due to the ease and increased availability of the digital images in public database. Most recent digital images of the celebrities available in the public

\section{Ifechukwuamaka Chinaka, Arun HS Kumar*}

Veterinary Biosciences, School of Veterinary Medicine, University College Dublin, Belfield, Dublin 4, IRELAND.

\section{Correspondence}

Dr. Arun HS Kumar, DVM, PhD.

School of Veterinary Medicine, University College Dublin, Belfield, Room 216, Dublin-04, IRELAND.

Ph.no: 00-353-17166230

Email: arun.kumar@ucd.ie

History

- Submission Date: 01-11-2019;

- Review completed: 21-11-2019;

- Accepted Date: 26-11-2019.

DOI : 10.5530/bems.5.2.8

Article Available online

http://www.bemsreports.org

Copyright

(C) 2019 Phcog.Net. This is an open-access article distributed under the terms of the Creative Commons Attribution 4.0 International license. 
database during the time of this study analysis (January to April 2019) were used. For the celebrities who succumbed to myocardial infarction the most recent digital image just prior to their month of death was used for the analysis. After a detailed background search a list of 48 celebrities (20 who succumbed to myocardial infarction (Cel-Mi) and 28 celebrities without any known health conditions (Cel-Li)) from different geographical locations were shortlisted for this study analysis. We limited the age group of celebrities included in this study to 25-80 years.

The digital images were analysed for Iris and pupil area and perimeter using NIH Image J software. ${ }^{24}$ Images with imperfect angle of the eye, where iris and pupil couldn't be clearly outlined were excluded from the analysis. Oval or free hand selection tools were used to outline and measure iris and pupil parameters. ${ }^{22,23}$ The data were transferred to a Microsoft Excel sheet for calculation of the iris to pupil area ratio and the data were analysed using GraphPad Prism software (Version 5) with significance accepted at $p<0.05$.

\section{RESULTS}

The mean age of the celebrities who succumbed to myocardial infarction (Cel-Mi) was $58.3 \pm 15.5$ years (range: 26 to 78 years) $(n=20)$, while the mean age of the celebrities in the healthy cohort (Cel-Li) was $45.5 \pm 16.4$ years (range 27 to 75 years) $(n=28)$.
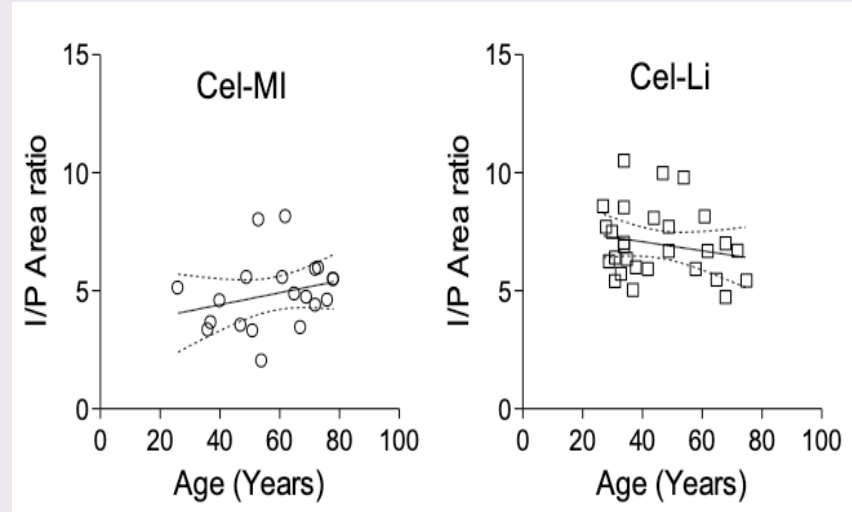

Figure 1: Iris/pupil (I/P) area ratio of celebrity cohort who succumbed to myocardial infraction (Cel-Ml; $n=20$ ) and healthy celebrities with no known health conditions (Cel-Li; $n=28$ ). Data is presented as Mean $\pm \mathrm{SD}$. *** $p<0.001$.

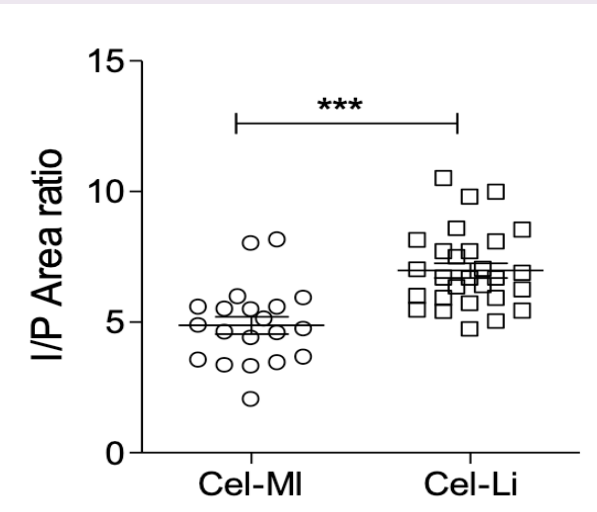

Figure 2: Correlation analysis of Iris/pupil (I/P) area ratio with age (Years) of celebrity cohort who succumbed to myocardial infraction (Cel-Ml; $n=20$ ) and healthy celebrities with no known health conditions (Cel-Li; $n=28)$.
The iris to pupil area ratio was significantly $(p<0.001)$ lower in the CelMi group compared to the Cel-Li group (Figure 1, Table 1). Suggesting a significantly dilated pupil in Cel-Mi group compared to Cel-Li group. Correlation analysis of the iris to pupil area ratio with age in both the cohorts indicated a differing outcome. In the Cel-Mi group the correlation of the iris to pupil area ratio with age was observed to be positive while this correlation was negative in Cel-Li group (Figure 2, Table 2). To assess the influence of age on the iris to pupil area ratio we performed a subgroup analysis in the both cohorts by dichotomising the age groups between $25-49$ and 50 to 80 years. While both cohorts showed a positive correction between iris to pupil area ratio and age (25-49 years), the correlation was different in the age range of 50 to 80 years (Table 2). The slope of the correlation line was steeper in the Cel-Mi group compared to Cel-Li group. Suggesting that the influence of age on iris to pupil area ratio was greater in the Cel-Mi group compared to Cel-Li group.

\section{DISCUSSION}

A fine balance between sympathetic and parasympathetic system very sensitively regulates the diameter of the pupil ${ }^{22,23}$ and perhaps the microvasculature..$^{13,15,16,20}$ Besides the autonomic system, several regulators of cardiac microvasculature physiology are also reported. ${ }^{14,19,21,25-27}$ Nevertheless the autonomic system remains the dominant regulator of microvascular function. ${ }^{20,28}$ Addressing the commonality between the regulation of pupil diameter and microvasculature by the autonomic system may be clinically useful as a non-invasive and perhaps sensitive index of predicting microvascular complications such as myocardial infraction. We have previously reported the evidence of such commonality in a primate and canine study cohorts wherein iris to pupil area ratio was demonstrated to be a reliable index of borderline changes in autonomic system activity. ${ }^{22,23}$ In this study we report evidence of commonality between the regulation of pupil diameter and microvasculature by the autonomic system in human subjects. Analysing the digital image of the celebrities who succumbed to myocardial infarction, we show here that their

Table 1: I/P area ratio values of the study subjects.

\begin{tabular}{|ccc|}
\hline & Cel-MI & Cel-Li \\
\hline Mean \pm SD & $4.875 \pm 1.506^{* * *}$ & $6.978 \pm 1.499$ \\
Range & 2.014 to 8.125 & 4.702 to 10.48 \\
Median & 4.791 & 6.666 \\
$25 \%$ percentile & 3.558 & 5.89 \\
$75 \%$ percentile & 5.557 & 7.96 \\
$* * *: p<0.001$ versus Cel-Li group & \\
Cel-MI: Celebrity cohort who succumbed to myocardial infraction $(n=20)$. \\
Cel-Li: healthy celebrities with no known health conditions $(n=28)$.
\end{tabular}

Table 2: Correlation coefficient of study subjects.

\begin{tabular}{|c|c|c|c|}
\hline & Cel-MI & Cel-Li & Combined \\
\hline Complete data & $0.06889^{*}$ & $0.03775 !$ & $0.05197^{!}$ \\
\hline b/w $25-49$ yrs & $0.00011^{\star}$ & $0.01614^{*}$ & $0.00248^{!}$ \\
\hline b/w 50 to 80 yrs & $0.00997^{\star}$ & $0.38731^{!}$ & $0.02168^{!}$ \\
\hline Slope & $0.02546 \pm 0.02206$ & $-0.01914 \pm 0.01895$ & $\begin{array}{c}-0.02512 \pm \\
0.01582\end{array}$ \\
\hline 1/Slope & 39.27 & -52.24 & -39.81 \\
\hline \multicolumn{4}{|c|}{${ }^{*}$ : Positive correlation } \\
\hline \multicolumn{4}{|c|}{ ! : Negative correlation } \\
\hline
\end{tabular}


iris to pupil area ratio was significantly lower compared to the healthy cohorts. Hence it is likely that coronary microvascular constriction triggered by over activity of sympathetic system, which can potentially lead to fatal myocardial infarction, is also reflected on collateral changes in the iris to pupil area ratio. Hence our study supports the hypothesis that iris to pupil area ratio can be useful as a non-invasive index of identifying patients at risk of suffering from myocardial infarction potentially due to over activity of sympathetic system. Another merit of iris to pupil area ratio as a non-invasive index of risk analysis is the feasibility to use it as a personalised monitoring tool in patient care.

The correlation analysis of iris to pupil area ratio with age showed a very interesting pattern. In the cohorts who succumbed to myocardial infarction the correction was positive, indicating a progressive increase in sympathetic activity with age. While in the healthy cohorts the correlation was negative, suggesting a slight dominance of parasympathetic system with increase in age. The difference observed in the correlation analysis of iris to pupil area ratio with age in both the cohorts was prominently evident in the age group of 50 to 80 years. While in both the cohorts the correlation analysis showed similar trends in the age group of 20-49 years. Several studies have previously reported changes in autonomic system with aging. ${ }^{29-32}$ Although the changes observed in autonomic system with aging is at a sub threshold level it is interesting to note that iris to pupil area ratio is sensitive enough to detect such changes, hence further validating the reliability of the this index in predicting clinical risk analysis associated with changes in autonomic system.

In conclusion the iris-pupil area ratio is a sensitive index of microvascular constriction induced by increased sympathetic activity and hence has merit to be used clinically as a non-invasive tool in identifying patients at risk of suffering from myocardial infarction.

\section{ACKNOWLEDGEMENT}

We acknowledge the support from University College Dublin-Seed funding/Output Based Research Support Scheme (AHSK) and Stemcology (AHSK).

\section{CONFLICT OF INTEREST}

The authors declare none.

\section{ABBREVIATIONS}

Cel-Mi: Celebrities who succumbed to myocardial infarction; Cel-Li: Celebrities without any known health conditions; NIH: National Institute of Health.

\section{REFERENCES}

1. Vogel B, et al. ST-segment elevation myocardial infarction. Nat Rev Dis Primers. 2019;5(1):39.

2. Inohara T, Endo A, Melloni C. Unmet Needs in Managing Myocardial Infarction in Patients With Malignancy. Front Cardiovasc Med. 2019;6:57.

3. D'Amario D, Vergallo R, Crea F. Predicting the future after acute myocardial infarction: A gaze into the crystal ball of gene expression profile. Int $\mathrm{J}$ Cardiol. 2018;254:47-8

4. Castro-Dominguez Y, Dharmarajan K, McNamara RL. Predicting death after acute myocardial infarction. Trends Cardiovasc Med. 2018;28(2):102-09.

5. Auffret $V$, et al. Predicting the development of in-hospital cardiogenic shock in patients with ST-segment elevation myocardial infarction treated by primary percutaneous coronary intervention: The ORBI risk score. Eur Heart J. 2018;39(22):2090-102.

6. Xiao Y, et al. Development and Validation of Risk Nomogram Model Predicting Coronary Microvascular Obstruction in Patients with ST-Segment Elevation
Myocardial Infarction (STEMI) Undergoing Primary Percutaneous Catheterization. Med Sci Monit. 2019;25:5864-77.

7. Pocock SJ, et al. Predicting risk of cardiovascular events 1 to 3 years postmyocardial infarction using a global registry. Clin Cardiol. 2019.

8. Huang J, Peng X, Fang Z, Hu X, Zhou S. Risk assessment model for predicting ventricular tachycardia or ventricular fibrillation in ST-segment elevation myocardial infarction patients who received primary percutaneous coronary intervention. Medicine. 2019;98:e14174.

9. Chen L, Han L, Luo J. Risk Factors for Predicting Mortality among Old Patients with Acute Myocardial Infarction during Hospitalization. Heart Surg Forum. 2019;22:E165-9

10. Hirji SA, et al. Predicting risk of cardiac events among ST-segment elevation myocardial infarction patients with conservatively managed non-infarct-related artery coronary artery disease: An analysis of the Duke Databank for Cardiovascular Disease. Am Heart J. 2017; 194:116-24.

11. Stone NJ, Lloyd-Jones DM. The Pooled Cohort Equations for Predicting Risk of Myocardial Infarction and Stroke: Validated in Representative Natural History Populations. Mayo Clin Proc. 2016;91(6):692-4.

12. Liu YH, et al. Comparison of Different Risk Scores for Predicting Contrast Induced Nephropathy and Outcomes After Primary Percutaneous Coronary Intervention in Patients With ST Elevation Myocardial Infarction. Am J Cardiol. 2016;117(12):1896-903.

13. D'Amario D, et al. Microvascular Dysfunction in Heart Failure With Preserved Ejection Fraction. Front Physiol. 2019;10:1347.

14. Wilson RF, Wyche K, Christensen BV, Zimmer S, Laxson DD. Effects of adenosine on human coronary arterial circulation. Circulation. 1990;82:1595-606.

15. Eleftheriadou I, et al. The association of diabetic microvascular and macrovascular disease with cutaneous circulation in patients with type 2 diabetes mellitus. J Diabetes Complications. 2019;33(2):165-70.

16. Gigante $A$, et al. Parasympathetic activity increases with digital microvascular damage and vascular endothelial growth factor in systemic sclerosis. Clin Exp Rheumatol. 2018;36(Suppl 113):24-7.

17. Cho YH, et al. Higher skin autofluorescence in young people with Type 1 diabetes and microvascular complications. Diabet Med. 2017;34(4):543-50.

18. Stoyneva ZB, Dermendjiev SM, Medjidieva DG, Vodenicharov VE. Microvascular reactivity during sympathetic stimulations in Raynaud's phenomenon. Int Angiol. 2016;35(6):593-8.

19. Bulte CS, et al. Myocardial Microvascular Responsiveness During Acute Cardiac Sympathectomy Induced by Thoracic Epidural Anesthesia. J Cardiothorac Vasc Anesth. 2017;31(1):134-41.

20. Nascimento AR, et al. Central Sympathetic Modulation Reverses Microvascular Alterations in a Rat Model of High-Fat Diet-Induced Metabolic Syndrome. Microcirculation. 2016;23(4):320-9

21. Dorbala $\mathrm{S}$, et al. Coronary microvasclar dysfunction is related to abnormalities in myocardial structure and function in cardiac amyloidosis. JACC Heart Fail. 2014;2(4):358-67.

22. Lin A, Maguire L, Kilroy D, Kumar AHS. The Utility of Iris-pupillary Area Ratio as a Non-invasive Index of Stress in Primates. BEMS Reports. 2018;4(1):20-2.

23. Lin A, Bourque C, Kilroy D, Kumar AHS. Evaluation of Iris-pupillary ratio as a non-invasive biomarker of higher sympathetic activity in hypertensive canines and felines. 33rd World Veterinary Congress (WVC) in Korea, August 2017.

24. Schneider CA, Rasband WS, Eliceiri KW. NIH Image to ImageJ: 25 years of image analysis. Nat Methods. 2012;9(7):671-5.

25. Daut J, Standen NB, Nelson MT. The role of the membrane potential of endothelial and smooth muscle cells in the regulation of coronary blood flow. J Cardiovasc Electrophysiol. 1994;5(2):154-81.

26. Sievert $\mathrm{H}$, et al. Effect of the calcium antagonist nisoldipine on coronary circulation and myocardial ischemia in temporary coronary occlusion. Z Kardiol. 1990;79(10):683-8.

27. Schwartz $\mathrm{H}$, et al. Temporal evolution of the human coronary collateral circulation after myocardial infarction. J Am Coll Cardiol. 1984;4(6):1088-93.

28. Heusch G, Thamer V. Significance of the sympathetic nervous system for the coronary circulation. Z Kardiol. 1984;73(9):543-51.

29. Parashar R, Amir M, Pakhare A, Rathi P, Chaudhary L. Age Related Changes in Autonomic Functions. J Clin Diagn Res. 2016;10(3):CC11-5

30. Sakatani K, Tanida M, Katsuyama M. Effects of aging on activity of the prefrontal cortex and autonomic nervous system during mental stress task. Adv Exp Med Biol. 2010;662:473-8.

31. Hotta H, Uchida S. Aging of the autonomic nervous system and possible improvements in autonomic activity using somatic afferent stimulation. Geriatr Gerontol Int. 2010;10(Suppl 1):S127-36.

32. Pfeifer MA, et al. Differential changes of autonomic nervous system function with age in man. Am J Med. 1983;75(2):249-58. 